\title{
Bullying escolar en niño como consecuencia de su estado de salud bucal: reporte de caso
}

\author{
Bullyng school in child as a result of oral health: case report \\ Gissela Gálvez-Cubas ${ }^{l, a}$, Daniella Céspedes-Martínez ${ }^{l, a}$, Franks Gamero-Castillo ${ }^{l, a}$, \\ Cecilia Tomás-De la Cruz ${ }^{1, a}$, María Elena Díaz-Pizán $n^{2, a, c, d}$
}

\section{RESUMEN}

El término bullying escolar, se refiere a todos los comportamientos agresivos y antisociales, incluyendo los conflictos interpersonales dentro de un ambiente escolar, teniendo como consecuencias en las víctimas: depresión, bajo rendimiento escolar y aislamiento social. El bullying escolar, es un fenómeno multifactorial, que se presenta principalmente en niños y adolescentes, y presenta múltiples factores causales, siendo uno de ellos el mal estado de salud bucal del niño. Se presenta el reporte de caso de un niño de 7 años 8 meses de edad, donde se describe la rehabilitación bucal por presentar múltiples lesiones de caries dental, alterando su apariencia física, motivo por el cual fue víctima de burlas en su centro educativo.

PALABRAS CLAVE: Acoso escolar, caries dental, rehabilitación bucal. (DECS, BIREME)

Facultad de Estomatología Roberto Beltrán, Universidad Peruana Cayetano Heredia. Lima, Perú.

Sociedad Peruana de Odontopediatría. Lima, Perú.

Cirujano Dentista.

Residente de Odontología Pediátrica.

Especialista en Odontología Pediátrica

Magister en Estomatología. 


\section{SUMMARY}

The term school bullying relates to all aggressive and antisocial behavior, including interpersonal conflicts within the school environment, having these consequences in the victims: depression, poor school performance and social isolation. The school bullying is a multifactorial phenomenon, which occurs mainly in children and adolescents, having multiple etiologic factors, being one them the poor oral health status of the child. This is a case report presentation of a 7 years 8 months male patient, which describes the oral rehabilitation of multiple carious lesions which affects his physical appearance making him a victim of mocking in his school.

\section{KEYWORDS: Bullying, dental caries, oral rehabilitation. (MeSH, NLM)}

\section{INTRODUCCIÓN}

El acoso escolar o bullying escolar en niños y adolescentes se ha convertido en un problema mundial y de preocupación para todos. Su prevalencia dentro de la escuela en los diferentes países oscila entre 5\% a $57 \%$, pero muy pocos casos son denunciados (1). El acoso escolar es un comportamiento agresivo llevado a cabo repetidamente en el tiempo, es una relación caracterizada por un desequilibrio de poder, a lo cual han denominado también bullying; y con el transcurrir de los años, parece ser común en niños y adolescentes $(1,2,3)$.

Existen 2 formas de manifestación del bullying (1, $3,4,5,6,7)$ :

a. Acoso directo, que incluye la agresión física (golpes, patadas), la agresión verbal (insultos, amenazas).

b. Acoso indirecto, que implica la manipulación de las relaciones sociales (chismes, rumores, exclusión social).

Los varones tienden a experimentar formas directas, mientras que las mujeres son más propensas a sufrir formas indirectas $(6,7)$.

La forma más común de acoso es la directa, mediante la agresión verbal, esta forma de acoso lleva el nombre-calling. Es común que se presente entre niños o adolescentes y puede ocurrir en cualquier ambiente, por lo general ocurre en el patio de recreo de la escuela. Otro de los lugres es el trayecto hacia la escuela $(1,2,5,8)$.
El bullying puede causar un impacto negativo en el niño a largo plazo, manifestando el niño: depresión, baja autoestima, problemas mentales y físicos, pobre rendimiento académico y como consecuencia más drástica una conducta delictiva $(3,7,9)$.

La manera de prevenir el bullying, va a depender del enfoque positivo de los padres en cuanto a las relaciones con sus hijos, este parece ser muy importante para el desarrollo equilibrado del niño. La asistencia a sus necesidades básicas, la seguridad y confianza que se le de, proporcionará al niño la confianza en sí mismo para responder adecuadamente a las dificultades que puedan surgir en la vida. Cuando hay un contacto limitado del padre y la madre, el niño suele ser más vulnerable al bullynig. Algunas causas más resaltantes de esto son: el divorcio, ausencia física del padre (muerte) o cuando los padres son indiferentes a la vida del niño $(8,11)$.

Los métodos de crianza y disciplina de los padres influyen en el comportamiento de los niños, se ha reportado que los niños que realizan bullying o son víctimas de esto, provienen de familias de modelo autoritario y dominante. Por lo tanto, ¿qué medidas o comportamientos deben adoptar los padres para evitar que sus hijos sean susceptibles a practicar o padecer de bullying? (8).

Los padres deben fomentar la autonomía de sus hijos, dejar que ellos tomen sus propias decisiones $\mathrm{y}$ regulen sus propias actividades de una manera responsable y bajo reglas justificadas implantadas por ellos; de esta manera, formar hijos con autoestima y carácter para enfrentar situaciones difíciles (11). 
La comunicación diaria entre padres e hijos, es la herramienta más importante y desempeña un papel crucial en el desarrollo mental y rendimiento personal del niño en formación (13).

El bullying puede ser consecuencia también del estado de salud bucal en niños o adolescentes, y es que las características dentales también contribuyen a los apodos, al acoso, y las burlas entre escolares (5, $13,14)$.

Al-Bitar y col. (1) sostienen, que la prevalencia de las burlas relacionadas con la apariencia dental es de $15 \%$ aproximadamente (4). Además, los comentarios con relación al estado dental serían más hirientes que los relacionados con otras características físicas tales como la altura o el peso $(1,4)$.

Generalmente, el apiñamiento de dientes, el overjet y/o overbite aumentados son causas de bullying, lo cual genera un impacto negativo en la autoestima y calidad de vida $(14,5,9)$. Sin embargo, cuando el niño inicia su tratamiento de ortodoncia, se reporta una menor frecuencia $(1,5,6,13,14)$.

No solo el presentar defectos de alineamiento puede ser causa de bullying; sino también la presencia de múltiples lesiones cariosas, que involucra la apariencia física de una persona. Esto también es causa de acoso, que dentro de un ámbito escolar va a traer como consecuencia: bajo rendimiento académico, aislamiento social y por ende un comportamiento diferente a sus pares (1).

El presente reporte describe un caso de bullying escolar a un niño de 7 años 8 meses de edad, debido a la presencia de múltiples lesiones cariosas, quien a causa de su apariencia y su salud bucal fue víctima de constantes burlas en su centro educativo.

\section{Reporte de caso}

Paciente niño de 7 años 8 meses de edad en ABEG, natural y procedente de la ciudad de Lima - Perú, acude al servicio de Odontología Pediátrica de la Clínica Estomatológica Central de la Facultad de Estomatología Roberto Beltrán de la Universidad Peruana Cayetano Heredia, acompañado de su padre, quien refiere que su niño necesita ayuda y tratamiento odontológico, porque sufría burlas en su centro educativo y tiene el apodo de "dientes de chocolate", debido al mal estado de su boca, motivo por el cual ha notado cambios en su estado de ánimo, en su comportamiento y en su rendimiento escolar.

Al examen clínico extraoral; se observa un niño de apariencia triste, temeroso, con presencia de ojeras y dificultad para sonreír (Figura 1).

Al examen clínico intraoral; se observa dentición mixta primera fase, mala higiene bucal, halitosis, inflamación en la encía adherida, presencia de múltiples lesiones de caries dental en pzas. 54,55,5 $2,51,61,62,64,65,75,75,84,85$, fístula a nivel de las pzas. 54 y 75, pólipo pulpar en la pza. 64 (Figura 2).

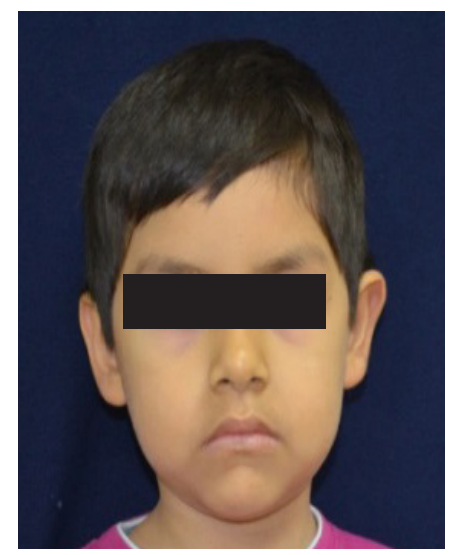

Figura 1. Apariencia extraoral: niño triste y con ojeras.
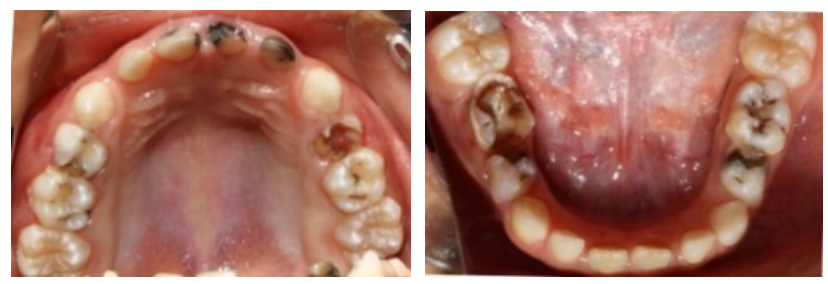

Figura 2. Fotos intraorales: múltiples lesiones cariosas.

Al examen radiográfico; se observa: Pza. 52 con lesión cariosa en zona distal y cara libre. Pza. 22 en evolución intra ósea, Nolla 5 y disto inclinada. Pza. 51 remanente coronario con lesión cariosa incisomesial e inciso-distal. Pza. 61 lesión cariosa en zona inciso-mesial y reabsorción radicular del tercio apical (Figura 3).

Pza. 64 lesión cariosa ocluso-distal (Figura 4), Pza. 75 lesión cariosa mesio-ocluso-distal amplia y profunda con compromiso pulpar, proceso osteolítico intra radicular compatible con absceso dento alveolar. 


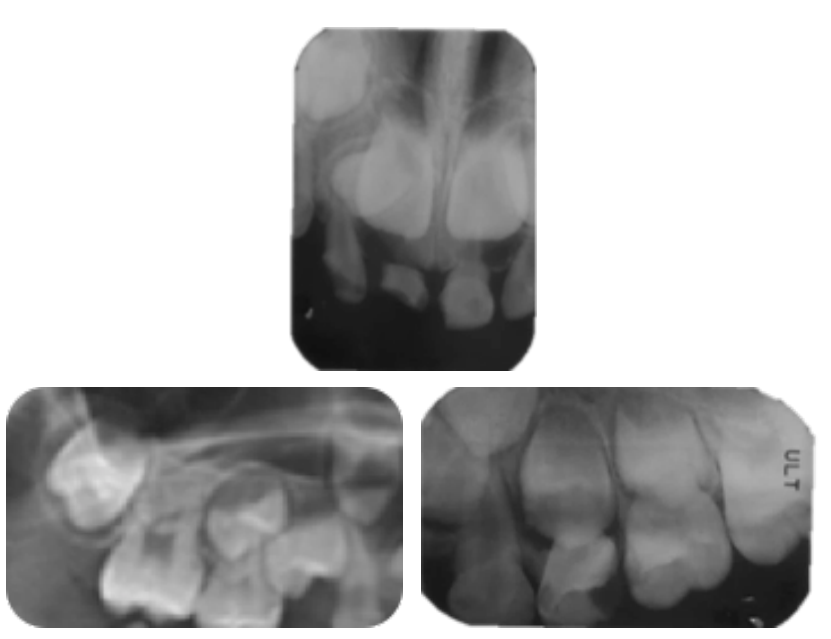

Figura 3. Piezas dentarias con destrucción coronaria.
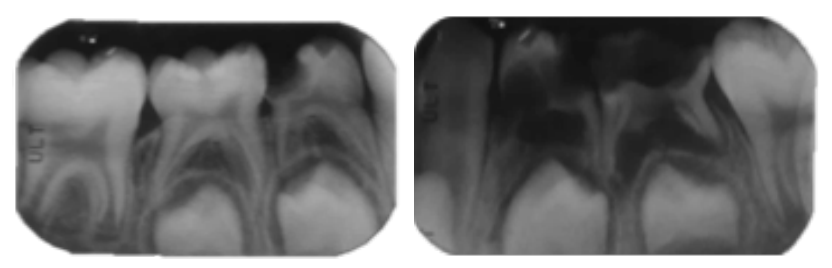

Figura 4. Pza. 75 lesión cariosa mesio-ocluso-distal amplia y profunda con compromiso pulpar, proceso osteolítico intra radicular compatible con absceso dento alveolar.

Pza. 74 lesión cariosa coronal, amplia y profunda con compromiso pulpar, reabsorción radicular interna raíz mesial, borramiento de la cortical de la cripta de la pza. 34, proceso osteolítico intra radicular compatible con absceso dento alveolar. Pza. 84 lesión cariosa ocluso-distal con probable compromiso pulpar. Pza. 85 lesión cariosa en cara libre (vestibular o lingual).

Radiografía panorámica: estadio Nolla 5 en Pzas.: 15, 25, 35, 45, 17, 27, 37, 47; estadio Nolla 6 en Pzas.: $12,22,14,24,34,44,13,23,33,43,17,27$; estadio Nolla 7 en Pzas.: 11, 21, 32, 42; estadio Nolla 8 en Pzas.: 16, 26, 36, 46, 31, 41. Representando una edad dentaria según Nolla de: 7 años de edad y de acuerdo al método de estimación de Dermillan de: 7 años y 5 meses de edad. (Figura 5).

Diagnóstico definitivo: luego de la evaluación clínica y los exámenes auxiliares necesarios se ha llega a el siguiente diagnóstico definitivo.

Tejidos blandos: gingivitis leve asociada a presencia de placa bacteriana, sin otro factor contribuyente.

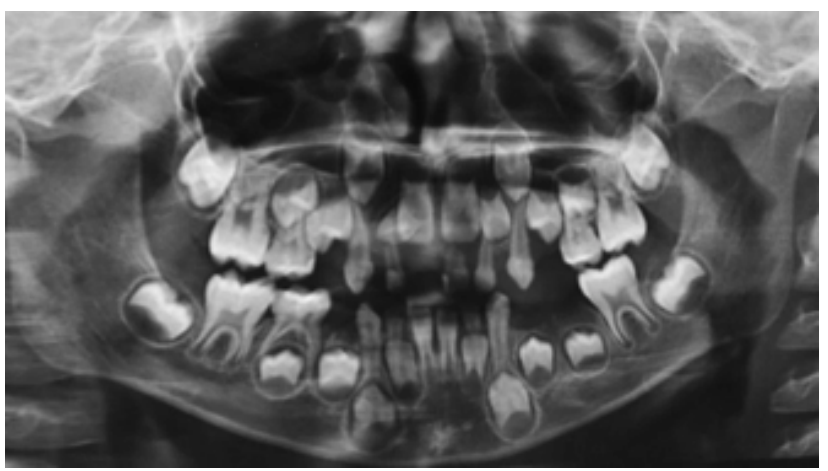

Figura 5. Radiografía Panorámica, representando una edad dentaria según Nolla de 7 años.

Tejidos duros: Caries dental con lesiones cavitadas en Pzas.: $52(\mathrm{~V}), 53(\mathrm{~V}), 55(\mathrm{M})(\mathrm{O})(\mathrm{P}), 61(\mathrm{~V})(\mathrm{M})$, 63(V), 65(M)(0)(P), $36(\mathrm{O}), \quad 85(\mathrm{M})(\mathrm{O})(\mathrm{V})$; pulpitis crónica hiperplásica en Pza.: 64, necrosis pulpar en Pzas.: 74, 75, 84 y remanente coronario en Pzas.: 51 y 54.

Riesgo estomatológico: alto.

\section{Conducta: receptiva.}

Tratamiento: El tratamiento se realizó en 4 fases para solucionar los problemas estéticos y funcionales devolviendóle al niño su salud bucal, mejorando la función masticatoria, la fonación, y la estética; y por ende, mejorando la relación social con sus compañeros de aula.

$1^{\circ}$ Fase de higiene: en la clínica se realizó, fisioterapia oral para incentivar al niño a una correcta técnica de cepillado, modificar costumbres de higiene bucal, y profilaxis. Se le indico pasta dental de 1100 ppm para su higiene bucal diaria. $2^{\circ}$ Fase preventiva: se aplicó flúor en barniz al 5\%, sellantes de fosas y fisuras en Pzas.: $16(\mathrm{O}), 26(\mathrm{O})$ y $46(\mathrm{O})$, exodoncias de las Pzas.: 51, 54, 64, 74 , 75,84 .

$3^{\circ}$ Fase correctiva: se realizó restauraciones con resina de las Pzas.: $52(\mathrm{~V}), 53(\mathrm{~V}), 55(\mathrm{M})(\mathrm{O})$ $(\mathrm{P}), 61(\mathrm{~V})(\mathrm{M}), 63(\mathrm{~V}), 65(\mathrm{M})(\mathrm{O})(\mathrm{P}), 63(\mathrm{~V}), 36$ $(\mathrm{O}), 85(\mathrm{M})(\mathrm{O})(\mathrm{V})$ y la confección de mantenedores de espacio removibles protésicos, para devolverle la función y estética a la cavidad bucal (Figura 5 y $6)$.

Después del tratamiento se pudo observar cambio de actitud del niño con su entorno, se observó un niño más alegre, que sonría sin vergüenza y temor 
a ser maltratado como lo era antes (Figura 7).

$4^{\circ}$ Fase de mantenimiento, se programaron controles clínicos periódicos, cada 2 meses.

Los tratamientos realizados mostraron mejoría en el paciente. El padre manifestó que el niño había mejorado las relaciones con sus compañeros, su rendimiento académico, y presentaba un mejor semblante.
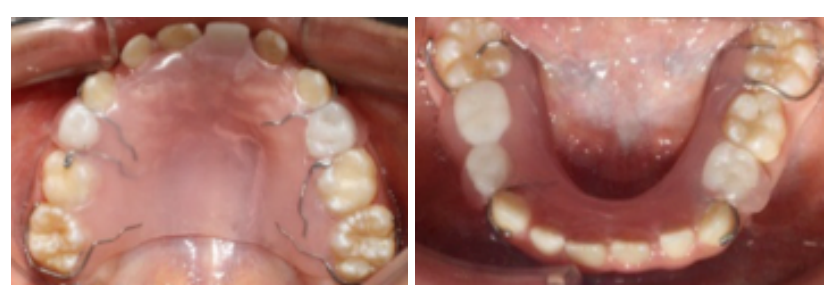

Figura 6. Rehabilitación integral de la boca del niño

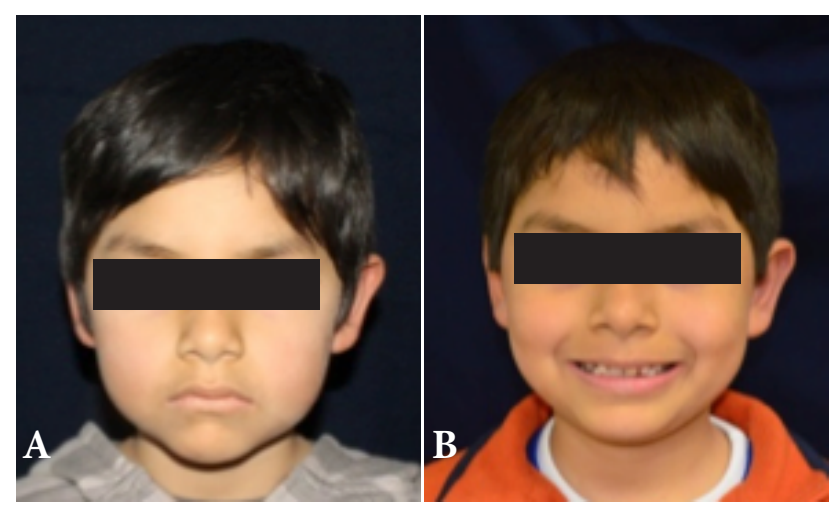

Figura 7. Fotos extraorales antes (A) y después (B) del tratamiento. Se puede observar el cambio de estado emocional.

\section{DISCUSIÓN}

Seehra y col. (6) refieren que presentar patrones dentales alterados aumenta el riesgo a burlas, lo que trae como consecuencia una interrupción de la normalidad del desarrollo psicológico del niño, que se confirma en el presente reporte de caso. El niño por presentar una alteración en su aspecto físico por el mal estado de sus piezas dentales, fue víctima de burlas por parte de sus compañeros, consiguiendo que se excluya del grupo $(6,10)$.

Queda demostrado que los niños son más propensos a sufrir burlas o intimidación en comparación con las personas adultas $(2,3,7)$. En cuanto a género, varios estudios refieren que son los varones más propensos al bullying directo, tal como queda demostrado en el presente caso, ya que el motivo de consulta básicamente fue por burlas verbales de sus compañeros, debido a su mal estado bucal $(8,10,13)$.

Los padres que muestran mayor dedicación en el cuidado de sus hijos, desarrollan en ellos hábitos para su cuidado y desarrollo personal, tales como: hábito de higiene bucal, responsabilidad; así como también, buena autoestima $(1,2,6)$. Todo lo contrario sucedió con el niño del presente reporte, dado que sus padres, trabajaban fuera de casa la mayor parte del día, mostrando descuido por su salud bucal, y tuvo como consecuencia una baja autoestima y depresión $(10,13,14,15)$.

La depresión es una consecuencia del bullying, originando en el niño el aislamiento de su entorno, dificultad en relacionarse, y como consecuencia estos niños buscan actividades sedentarias, que requiere una relación social mínima, actividades en solitario como: ver televisión, videojuegos o permanecer durante horas delante de la computadora. Manifestaciones que alertan al entorno familiar y a los padres a percibir que sus niños son víctimas de bullying $(5,9)$.

Dentro del ámbito escolar, los niños víctimas de acoso, suelen bajar su rendimiento académico, condición que se pudo apreciar en el presente reporte. La apariencia estética se ve relacionada al estado de la salud bucal, y es otra razón que da lugar a burlas e intimidación, siendo la odontología restauradora una alternativa para la solución inmediata del problema, como fue en el caso expuesto. Se devolvió la estética y funcionalidad, mediante la colocación de mantenedores protésicos, recuperando la confianza en sí mismo e integrándose a a su grupo social $(8,10)$.

Zaidy col.(10)demostraron que losniños que fueron sometidos a tratamiento de ortodoncia para corregir mal posiciones dentarias, tuvieron como resultado que las burlas hacia ellos fueron disminuyendo conforme su tratamiento iba evolucionando y sus problemas dentarios progresivamente se iban corrigiendo. En el presente caso se pudo observar que después del tratamiento rehabilitador se solucionó de manera inmediata el problema, disminuyendo las burlas que sufría el niño en su centro educativo e integrándose a sus actividades cotidianas (10). 


\section{CONCLUSIONES}

Existe una relación directa entre salud bucal deficiente con problemas estéticos y acoso escolar.

El odontólogo pediátrico no solo debe enfocarse en la rehabilitación de la boca del niño, sino también en la rehabilitación integral de la salud física y mental del niño.

El tratamiento realizado devolvió al niño la confianza en sí mismo; mejorando su apariencia física, función masticatoria, fonación y previniendo la aparición de maloclusiones futuras.

\section{Correspondencia}

\section{Gissela Gálvez Cubas}

Jhon Kenedy 204 Dpto. 101, Pueblo Libre. Lima, Perú. Correo electrónico: gissela.galvez.c@upch.pe

\section{REFERENCIAS BIBLIOGRÁFICAS}

1. Al-Bitar, Al-Omari, Sonbol, Al-Ahmad, and Cunninghamd. Bullying among Jordanian schoolchildren, its effects on school performance and the contribution of general physical and dentofacial features. Am J Orthod Dentofacial Orthop. 2013; 144:872-8.

2. Seehra, Newton and DiBiase. Bullying in schoolchildren - its relationship to dental appearance and psychosocial implications: an update for GDPs. British Dental Journal. 2011; 210(9):411-5.

3. Wang, Iannotti and Nansel. School bullying among adolescents in the United States: physical, verbal, relational, and cyber. Journal of Adolescent Health. 2009; 45 (4):368 -75.

4. Papanikolaou, Chatzikosma, Kleio. Bullying at school: the role of family. Procedia - social and behavioral sciences. 2011; 29:433-42.

5. Brito and Oliveira. Bullying and self-esteem in adolescents from public schools. J Pediatr (Rio J). 2013; 89(6):601-7.
6. Perlus, Brooks-Russell, Wang, Iannotti. Trends in bullying, physical fighting, and weapon carrying among 6th- Through 1Oth-Grade Students From 1998 to 2010: findings from a national study. Am J Public Health. 2014. 104(6): 1100-1106

7. Almeida, Cabral-Xavier, Paiva and Leite-Cavalcanti. Prevalência e Tipos de Bullying em Escolares Brasileiros de 13 a 17 años. Rev. Salud Pública. 2014; 16 (2):173-83.

8. Seehra, Newton and DiBiase. Interceptive orthodontic treatment in bullied adolescents and its impact on selfesteem and oral-health-related quality of life. Eur J Orthod. 2013; 35 (5):615-21.

9. Rech, Halpern, Tedesco and Santos. Prevalence and characteristics of victims and perpetrators of bullying. J Pediatr (Rio J). 2013; 89(2):164-70.

10.Zakeria and Karimpour. Parenting styles and selfesteem. Procedia - social and sehavioral sciences. 2011; 29:758- 61 .

11. Narjes Khatoon Zabihi Hesari, Elahe Hejazi. The mediating role of self esteem in the relationship between the authoritative parenting style and aggression. Procedia - social and behavioral sciences. 2011; 30:1724-30.

11. Cassiani, Gómez, Cubides, Hernández. Prevalencia de bullying y factores relacionados en estudiantes de bachillerato de una institución educativa de Cali, Colombia, 2011. Rev. salud pública. 2014; 16(1):14-26.

12. Keshavarz and Baharudin. The moderating role of gender on the relationships between perceived paternal parenting style, locus of control and self-efficacy. Procedia-social and behavioral science. 2012; 32:63-8.

13. Rothon, Head, Klineberg and Stansfeld. Can social support protect bullied adolescents from adverse outcomes? A prospective study on the effects of bullying on the educational achievement and mental health of adolescents at secondary schools in East London. Journal of Adolescence. 2011; 34 (3): 579-88.

Recibido: 07/04/2015

Aceptado: 05/05/2015 